\title{
RCS analysis on different targets and bistatic angles using LTE frequency
}

\begin{abstract}
Moving target detection and location are a function of dependent bistatic Radar Cross Section (RCS) and radar design parameters which in our experimental study used long-term evolution (LTE) signal as a source for passive bistatic radar (PBR). Moving target also can be classified in positions of different bistatic radar angles using conventional processing approaches which we performed a simulation using Computer Simulation Technology (CST) Microwave studio. The target bistatic radar cross-section, sb will give a realistic calculation on PBR performance with the requirement of complete treatment. The targets used are Toyota Rush (SUV) and Proton Exora (compact MPV) as a moving target had been designed based on the actual size to observe the performance of RCS due to the changing of bistatic angle between transmitter and receiver. The frequency transmit signal from LTE based station is $2.6 \mathrm{GHz}$, far-field conditions and the material used for the moving target is perfect electrical conductor. Simulation used different bistatic angles which are $59^{\circ}$ and $90^{\circ}$ of plane wave propagation. The simulation results show that largest area of moving target had better outcome that reliable with Babinet's principle, which declares a target of physical cross-sectional area is proportionate to RCS. The variation of RCS also depends on the shape of moving target, size of moving target and angles of plane wave. This might improve the sensitivity elevation targets with an adjustment of receiver angle to the target and transmitter for a better RCS performance.
\end{abstract}

Keyword: RCS analysis; Bistatic angles; LTE frequency 